\title{
Association of GRACE Risk Score with Angiographic Severity of Coronary Artery Disease in patients with ST Elevation Myocardial Infarction
}

\author{
Tapash Saha ${ }^{1}$, Md. Khalequzzaman², Md. Abdul Kader Akanda², Simu Saha ${ }^{3}$, Asif Zaman Tushar², \\ Rashid Ahmed ${ }^{4}$, Gouranga Kumar Saha ${ }^{2}$, Mohammad Ullah ${ }^{2}$ \\ ${ }^{1}$ Upazila Health Complex, Delduar, Tangail, ${ }^{2}$ Department of Cardiology, \\ NICVD, ${ }^{3}$ International Medical College \& Hospital, Tongi, ${ }^{4}$ Upazila Health Complex, Nababgonj
}

\begin{abstract}
Key Words :
Risk

stratification, GRACE risk

Background: Clinical guidelines recommend that optimal management of acute coronary syndrome should include patient risk stratification. Predicting the anatomical extension of coronary artery disease is also potentially useful for clinical decision. The objective of our study is to determine whether the GRACE risk score correlates with the angiographic extent and severity of coronary artery disease in patients with ST elevation myocardial infarction.
\end{abstract}

Abstract: score, Coronary artery disease severity, Gensini score

Methodology: 50 patients diagnosed with Acute Myocardial Infarction were included as sample by purposive sampling method. GRACE risk score for each patient was calculated and the patients were divided into groups according to the GRACE risk score: low risk (<108); intermediate risk (109-140). The severity of the coronary artery disease was assessed by vessel score and Gensini score. Relation between Grace score and Gensini score was evaluated.

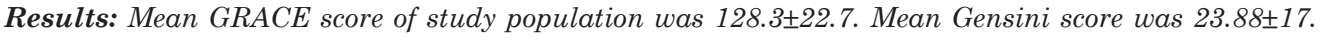

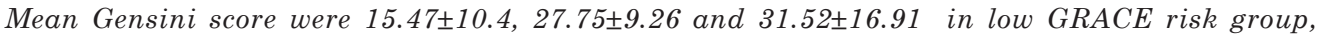
intermediate group and high risk group respectively and the difference of mean Gensini score was statistically significant $(p=0.006)$. In our study correlation co-efficient between GRACE risk score and Gensini score was $r=0.17(p=0.04)$. Multiple regression analysis showed that age more than 50 years $(p=0.02)$, ST segment deviation $(p=0.01)$, smoking $(p=0.02)$, hypertension $(p=0.01)$ were able to independently predict patients with severe CAD.

Conclusion: Our study demonstrates that the GRACE risk score carries a significant positive correlation with the coronary artery disease severity in patients with STEMI.

(Cardiovasc.j. 2015; 8(1): 30-34)

\section{Introduction:}

Coronary artery disease (CAD) is now the leading cause of death worldwide. Acute coronary syndrome (ACS) is a unifying term representing a common end result, acute myocardial ischemia and encompasses acute myocardial infarction (AMI)resulting in ST-segment elevation or non-STsegment elevation and unstable angina.The spectrum of ACS consists of a heterogeneous group of patients with considerable variation in clinical outcome. Clinical guidelines recommend that optimal management of ACS should include early, individualized patient risk stratification which is important for determining the prognosis, planning and the course of treatment and providing information for the patient relatives. ${ }^{1,2}$ The use of multivariate risk score models has been shown to represent the most accurate way to predict risk, being superior to the subjective clinical impression.

A number of risk scores have been developed to predict short and midterm outcomes in patients with ACS such as Framingham Risk score, Thrombolysis In Myocardial Infarction (TIMI) score and Global Registry of Acute Coronary Events (GRACE) score. ${ }^{3}$

The GRACE risk prediction model was developed from an earlier cohort of GRACE patients and has been extensively validated and shown to be a strong predictor of in hospital mortality across the

Address of Correspondence: Dr. Tapash Saha, Upazila Health Complex, Delduar, Tangail,Bangladesh. email:tapashsimu@gmail.com 
spectrum. The components of the GRACE risk score are age, heart rate, systolic blood pressure, Killip class, cardiac arrest, ST-segment deviation, serum creatinine, and initial cardiac biomarker status.

Although GRACE risk score is an important and validated tool for assessing prognosis but also its association with the extent of coronary artery disease is limited. In addition to prognostic assessment, predicting the anatomical extension of coronary artery disease is potentially useful for clinical decision. The objective of our study is to determine whether the GRACE risk score correlates with the angiographic extent and severity of coronary artery disease in patients with ST elevation myocardial infarction (STEMI) undergoing coronary angiography.

\section{Methodology:}

It was a cross sectional observational study. Study was conducted in the Department of Cardiology, National Institute of Cardiovascular Diseases, Dhaka between March 2013 to December 2013.All patients diagnosed with Acute Myocardial Infarction following $3^{\text {rd }}$ universal definition of Myocardial Infarction underwent coronary angiography during index hospitalization were the study population. Among them 50 patients were included by purposive sampling following the inclusion criteriaSTEMI who admitted within 12 hours of onset of chest pain. However, patients diagnosed with unstable angina valvular, congenital heart disease and cardiomyopathy, myocarditis or pericarditis, moderate to severe heart failure, a major non cardiovascular disorder and history of prior percutaneous coronary intervention or CABG were excluded.

Informed written consent was taken from each patient before enrollment. Evaluation of patients by taking history, clinical examination and data was recorded in a pre-designed structured questionere. GRACE risk score for each patient was calculated by using the online GRACE risk calculator. The patients were divided into groups according to the GRACE risk score: low risk $(<108)$; intermediate risk (109-140); and high risk (>140). ${ }^{4}$

Coronary angiogram was done after appropriate patient preparation. The severity of the $\mathrm{CAD}$ was assessed by vessel score and Gensini score.
Vessel score: Significant coronary artery disease was defined as $>70 \%$ stenosis in any of the three major epicardial coronary arteries or a left main coronary artery stenosis $>50 \%$. Extent of CAD was defined as significant single, two or three vessel CAD. ${ }^{5}$ Score ranges from 0 to 3 , depending on the number of vessel involve.

Gensini Score: A nonlinear score is assigned to each lesion based on the severity of stenosis as indicated by the reduction of lumen diameter. A multiplier was applied to each lesion score depending on the functional significance of the area supplied by that segment. The final Gensini score was the sum of the lesion scores. ${ }^{6}$ Gensini score value 36 was chosen as an appropriate cut-off value and patients were divided into 2 groups, those with a Gensini score $\leq 36$ points (absent or mild coronary atherosclerosis), and those with a Gensini score $>36$ points (medium to severe coronary atherosclerosis). ${ }^{7}$

Statistical analyses: The numerical data obtained from the study were analyzed and significance of differences were estimated by using statistical methods. The SPSS Statistical Software (16.0 version, SPSS Inc., Chicago, Illinois, USA) was used for data analysis. Continuous variables were expressed in mean \& standard deviation and categorical variables as frequency and percentage. Quantitative variables were analyzed by Student's t-test and ANOVA and Categorical variables were analyzed by chi-square test. To test association between GRACE risk score and coronary artery disease severity score Pearson's correlation test used. $\mathrm{P}$ value of less than 0.05 was considered as significant.

\section{Results:}

A total of 50 patients were included in the study. The average age of the patients was $50.7 \pm 8.7$. Majority of the study participants were male (69\%) and $31 \%$ were female. A number of patients were hypertensive (72\%), smoking habit was found in $62 \%$ patients. Chewing tobacco was observed in $24 \%$ patients. $32 \%$ patients had diabetes mellitus. Dyslipidemia and family history of CAD were observed in $50 \%$ and $36 \%$ patients respectively. Mean GRACE score was $128.3 \pm 22.7$ (Fig.1) Mean Gensini score was $23.88 \pm 17$. 


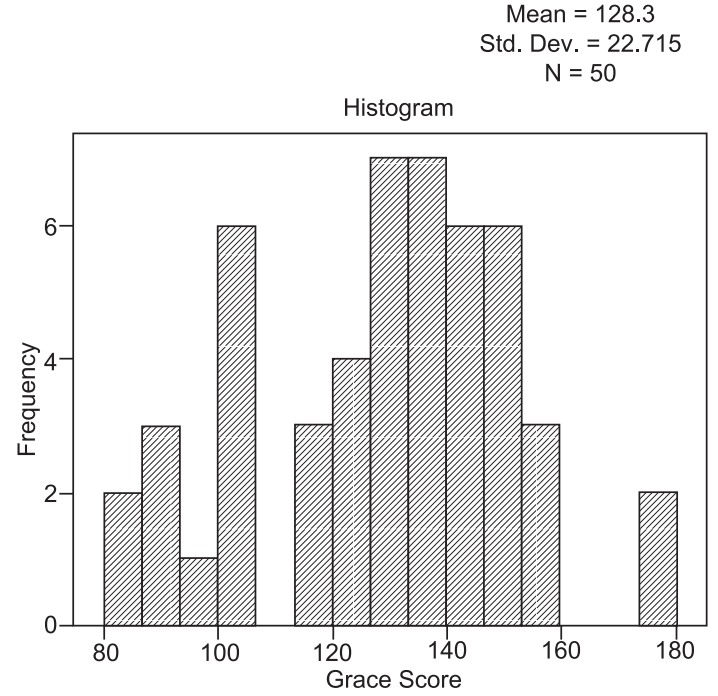

Fig.-1: Distribution of the study population according to GRACE score $(n=50)$.

Table-I

Association between GRACE Risk score and Gensini score $(n=50)$.

\begin{tabular}{llrl}
\hline GRACE Risk Score & \multicolumn{2}{c}{ Gensini Score } & P value \\
\cline { 2 - 3 } & Mean & SD & \\
\hline Low $(<108) \mathrm{n}=12$ & 15.47 & 10.40 & \\
Intermediate & 27.75 & 9.26 & $0.006^{\mathrm{s}}$ \\
$\begin{array}{l}\text { (109-140) } \mathrm{n}=21 \\
\text { High }(>140) \mathrm{n}=17\end{array}$ & 31.52 & 16.91 & \\
\hline
\end{tabular}

$\mathrm{s}=$ Significant, $\mathrm{p}$ value reached from ANOVA test.

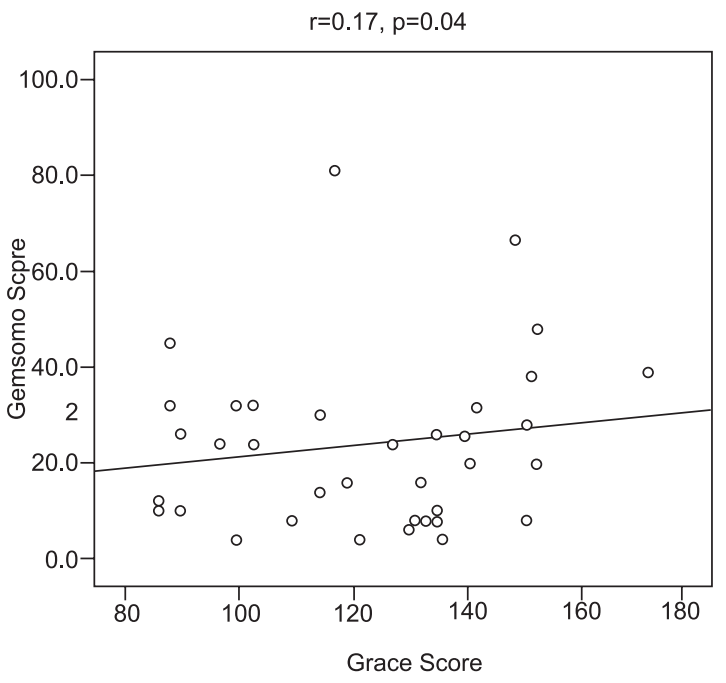

Fig.-2: Pearson's correlation between GRCAE and Gensini score.
Mean Gensini score were 15.47 $\pm 10.4,27.75 \pm 9.26$ and $31.52 \pm 16.91$ in low GRACE risk group, intermediate group and high risk group respectively and the difference of mean Gensini score was statistically significant $(\mathrm{p}=0.006)$. It can be said that there were tendency towards greater coronary artery disease extension according to GRACE tertile and it reached statistical significance $(p=0.006)$. So our findings are consistent with existing studies. ${ }^{8,9}$

In our study correlation co-efficient between GRACE risk score and Gensini score was $\mathrm{r}=0.17$ $(p=0.04)$. We found that Gensini score was positively and significantly associated with the GRACE score (Fig 2). With the increased GRACE score, Gensini score increases demonstrating more severe coronary atherosclerosis. This was in agreement with the previous observations. 8,9

Multiple regression analysis considering $\mathrm{CAD}$ as a dependent variable showed that after adjusting age, smoking, hypertension, dyslipidemia and family history of premature CAD and other variables of GRACE risk score, age more than 50 years $(\mathrm{p}=0.02)$, ST segment deviation $(\mathrm{p}=0.01)$, smoking $(p=0.02)$, hypertension $(p=0.01)$ were able to independently predict patients with severe CAD. These findings were supported by several other studies ${ }^{9-10}$. In the present study, CAD risk factors were not included in GRACE score such as hypertension and smoking were found to be correlated with Gensini score.

\section{Discussion:}

The objective of our study is to determine whether the GRACE risk score correlates with the angiographic extent and severity of CAD in patients with STEMI. Risk stratification is synonymous with prognosis determination. It is important during hospitalization and after discharge. Use of the GRACE score for stratifying risk in ACS was recommended by clinical practice guidelines. Management decisions in ACS should be based on a rapid and accurate assessment of risk. Patients who have the highest risk will have the poorest prognosis. The GRACE score provides an estimate of the probability of death within 6 months after hospital discharge in patients with ACS. ${ }^{3}$ Several studies have defined the relationship between coronary artery risk factors and severity of coronary artery lesions. Though most of these 
studies were on NSTEMI subset of ACS patients, the diagnostic prediction was similar for the entire ACS population. Regarding all this, this was a unique study describing the association between the GRACE score and coronary anatomy using Gensini Score.

Some risk factors may influence the development of coronary atherosclerosis at different levels of its evolution. There are different opinions about which stage of atherosclerosis is affected by diabetes mellitus and hypertension. Although some studies suggesting that diabetes mellitus and hypertension may affect early stages of atherosclerosis, other results do not support that idea. ${ }^{11}$ Additionally, renal function is also an important predictor of the presence and severity of angiographic CAD and creatinine level has an incremental value over traditional CAD risk factors. ${ }^{12}$ The increased prevalence of atherosclerotic coronary stenoses with the progression of age in both sexes has also been reported before in autopsy studies. The rate of this increase was, however, more prominent in men between 30 and 49 years of age. Recent data demonstrated a significant positive correlation between coronary angiographic scores and age, creatinine level, DM, and smoking, but not with blood pressure. ${ }^{13}$ In the present study, CAD risk factors not included in GRACE score such as hypertension and smoking were found to be correlated with Gensini score, but diabetes mellitus and creatinine level were not

In this study, coronary anatomy was described as a quantitative variable (Gensini score) and as a categorical variable (vessel score). A statistically significant correlation of the coronary anatomy with both scores was observed. The prognosis of coronary artery disease is known to be directly influenced by the extension of arterial impairment. ${ }^{14}$ GRACE risk score is used commonly for the clinical decision of patients with ACS, aiming at defining the aggressiveness of the antithrombotic therapy and the either invasive or selective strategy of stratification. ${ }^{4,15}$ That use is based on the established prognostic value of the score and on the relationship between baseline risk and the magnitude of the benefit of certain strategies. We should recognize that risk scores would be more useful if they could predict coronary anatomy. This is due to the fact that the invasive strategy, applied to patients at medium to high risk according to those scores, is completed with a procedure of revascularization of the culprit artery. If the scores could better identify the presence of obstructive disease, they would identify patients to whom the invasive strategy would be more useful. Another example of usefulness would be the prediction of patients with extensive disease, preventing the administration of certain antiplatelet agents that would increase their risk of bleeding in case of an occasional early surgical treatment. Regarding the TIMI score, four studies have assessed that question. All have reported a positive association between score value and coronary artery disease extension. Our data confirm the association with disease extension, but demonstrate that it is a weak predictor of disease severity.

There are some facts to be considered which might affect the results. Firstly, sample size was small in number to generalize the results because of invasive nature of the study and it was conducted in a single centre. Then, sampling method was not random rather purposive, so there is risk of selection bias. Finally, coronary angiography was assessed by visual observation, so there was every chance of interobserver variation.

\section{Study Limitations:}

There are some facts to be considered which might affect the results. Firstly, sample size wassmall in number to generalize the results because of invasive nature of the study and it was conducted in a single centre. Then, sampling method was not random rather purposive, so there is risk of selection bias. Finally, coronary angiography was assessed by visual observation, so there was every chance of interobserver variation.

\section{Conclusion:}

In conclusion, our study demonstrates that the GRACE risk score carries a significant positive correlation with the coronary artery disease severity in patients with STEMI. From this study it may be recommended that GRACE risk score can be used in patients with STEMI in the emergency department, coronary care unit or post coronary care unit at bed side to predict the prognosis and extent of coronary artery disease 
and provide information to the relatives of the patients about the planning of further management and their motivation for early intervention. In future multicentre, prospective study needed to assess the association between GRACE risk score and severity of coronary artery disease.

\section{Conflict of Interest - None.}

\section{References:}

1. Anderson JL, Adams CD, Antman EM, Bridges CR, Califf RM, Donald E et al. ACC/AHA 2007 Guidelines for the Management of Patients With Unstable Angina/ Non-ST-Elevation Myocardial Infarction : A Report of the American College of Cardiology/American Heart Association Task Force on Practice Guidelines. Circulation 2007; 116:e148-e304.

2. White HD, Wong CK. Risk stratification and treatment benefits in patients with non-ST-elevation acute coronary syndromes Eur Heart J 2002; 23(3):187-191.

3. Eagle KA, Lim MJ, Dabbous OH, Pieper KS, Goldberg RJ, Werf FVd et al. A Validated Prediction Model for All Forms of Acute Coronary Syndrome Estimating the Risk of 6-Month Post discharge Death in an International Registry. JAMA 2004; 291(22):2727-2733.

4. Hamm CW, Bassand J-P, Agewall S, Bax J, Boersma E, Bueno $\mathrm{H}$ et al. ESC Guidelines for the management of acute coronary syndromes in patients presenting without persistent ST-segment elevation The Task Force for the management of acute coronary syndromes (ACS) in patients presenting without persistent STsegment elevation of the European Society of Cardiology (ESC). Eur Heart J 2011; 32:2999-3054.

5. Chaitman BR, Bourassa MG, Davis K, Rogers WJ, Tyras $\mathrm{DH}$, Berger $\mathrm{R}$ et al. Angiographic prevalence of highrisk coronary artery disease in patient subsets (CASS). Circulation 1981;64: 360-367.

6. Gensini GG. A more meaningful scoring system for determining the severity of coronary heart disease. $\mathrm{Am}$ J Cardiol 1983;51: 606.
7. Ling S, Shu-zheng L. Association between non-alcoholic fatty liver disease and coronary artery disease severity. Chin Med J 2011;124:867-872.

8. Barbosa CE, Viana M, Brito M, Sabino M, Garcia G, Maraux M et al. Accuracy of the GRACE and TIMI Scores in Predicting the Angiographic Severity of Acute Coronary Syndrome. Arquivos Brasileiros de Cardiologia 2012;99: 818-824.

9. Cakar MA, Sahinkus S, Aydin E, Vatan MB, Keser N, Akdemir $\mathrm{R}$ et al. Relation between the GRACE score and severity of atherosclerosis in acute coronary syndrome. Journal of Cardiology 2014;63:24-28.

10. Latheef K, Praveen M, Vanajakshamma V, Rajasekhar D. Correlation of coronary artery disease angiographic severity with intima-media thickness of carotid artery. Journal of Indian College of Cardiology; 2(4):144-149.

11. Kasaoka S, Okuda F, Satoh A, Miura T, Kohno M, Fujii $\mathrm{T}$ et al. Effect of coronary risk factors on coronary angiographic morphology in patients with ischemic heart disease. Jpn Circ J 1997;61:390-395.

12. Saha JN. Association of impaired renal function with severity of coronary artery disease in patients with chronic stable angina University of Dhaka; 2014.

13. Mi SH, Su G, Li Z, Yang HX, Zheng H, Tao H et al. Comparison of glycemic variability and glycated hemoglobin as risk factors of coronary artery disease in patients with undiagnosed diabetes. Chin Med J (Engl) 2012;125:38-43.

14. Huang G, Zhao J-l, Du H, Lan X-b, Yin Y-h. Coronary Score Adds Prognostic Information for Patients With Acute Coronary Syndrome. Circ J 2010; 74: 490- 495.

15. Anderson JL, Adams CD, Antman EM, Bridges CR, Califf RM, Casey DE Jr et al. 2011 ACCF/AHA Focused Update Incorporated Into the ACC/AHA 2007 Guidelines for the Management of Patients With Unstable Angina/ Non-ST-Elevation Myocardial Infarction: a report of the American College of Cardiology Foundation/ American Heart Association Task Force on Practice Guidelines. Circulation 2011;123(18):e426-e579. 